\title{
OTIOTOMICS
}

Revista de economía, empresa y sociedad

Dossier «Marketing digital: revolucionando el consumo y la sociedad»

USO DE NUEVAS TECNOLOGÍAS

\section{La investigación de marketing con dispositivos móviles: un lento despertar}

\section{Garmen Pacheco Bernal}

Directora del grado en Marketing e investigación de mercados (UOC)

RESUMEN De la misma forma que la revolución móvil ha tenido un profundo impacto en la sociedad, ha creado nuevas actividades económicas y ha modificado las existentes, la industria de la investigación de marketing no se ha quedado al margen de todas estas transformaciones. Como veremos a continuación, las tecnologías móviles han representado nuevos retos y oportunidades para los profesionales del sector, generando nuevas formas de recoger datos de los consumidores que participan en investigaciones de mercado. Sin embargo, y a pesar de las ventajas que supone el uso de metodologías móviles para la industria, su adopción y difusión como técnica de recogida de información sigue siendo bastante limitada. En este artículo se recogen también los datos de uso de metodologías de investigación publicados por el sector y se identifican los principales factores que han influido en la adopción de la investigación de marketing con dispositivos móviles, ya sea incentivando o limitando su utilización.

PALABRAS CLAVE investigación de mercados; dispositivos móviles; investigación móvil; investigación en línea; adopción; TOE; ESOMAR; AEDEMO

\section{Mobile Market research: a slow awakening}

ABSTRACT Just as the mobile revolution has had a profound impact on society, has created new economic activities and modified existing ones, the marketing research industry has not remained at the margin of all these transformations. As we will see below, mobile technologies have represented new challenges and opportunities for the professionals in the sector, creating new ways to collect data from consumers who participate in market research studies. However, in spite of the advantages that the use of mobile methodologies involve for industry, their adoption and diffusion as a technique to collect information still remains fairly limited. This article includes the data on research methodologies used by the sector and identify the main factors that have influenced the adoption of mobile market research, whether to incentivise or limit their use.

KEYWORDS market research; mobile devices; mobile research; on-line research; adoption; TOE; ESOMAR; AEDEMO 


\section{Introducción}

La investigación de marketing con dispositivos móviles ha sido considerada durante muchos años the next big thing por parte del sector (Maxl, 2009; Poynter et al., 2014). Desde los primeros años del nuevo milenio, académicos y profesionales pronosticaban que las metodologías móviles de recogida de información iban a reemplazar las metodologías online, las cuales, encabezadas por las encuestas en línea, lideran desde hace años los primeros puestos en el ranking de técnicas empleadas en la recogida de información en las investigaciones de mercado. Como veremos más adelante, esto no está siendo así, y aunque la investigación móvil se ha ido aplicando, su uso no ha logrado desbancar a la investigación online.

¿Qué es exactamente la investigación de marketing con dispositivos móviles? Englobaría el conjunto de técnicas o metodologías de investigación de mercados en línea a través de las cuales se recaba información de los participantes mediante dispositivos electrónicos portátiles que están conectados a internet, entre estos los teléfonos móviles inteligentes, los tabléfonos, ${ }^{1}$ las tabletas o las tecnologías ponibles. ${ }^{2}$ Estamos hablando de:

- Investigaciones cuantitativas en línea, en las cuales los participantes responden encuestas en sus dispositivos móviles o en las que una aplicación descargada en sus dispositivos móviles recoge información sobre su entorno, lo que sería recogida pasiva de datos; esto es, datos recogidos desde los dispositivos móviles sin la intervención activa de los participantes.

- Investigaciones cualitativas en línea, en las que se participa a través de dispositivos móviles (por ejemplo, en una reunión de grupo o en una comunidad de investigación en línea), o en las que se recogen datos de los participantes (fotos, vídeos, etc.), como en los estudios etnográficos.

- También se suelen incluir las encuestas personales en las que se recogen las respuestas mediante dispositivos móviles (MCAPI [mobile computer-aided personal interviewing]) y las encuestas telefónicas en las que los entrevistados responden a través de sus teléfonos móviles (MCATI [mobile computer-aided telephone interviewing]).

Ha sido inevitable que el sector se interesara por las metodologías móviles, dada la gran popularidad y penetración de los dispositivos móviles, principalmente de los teléfonos móviles inteligentes, entre la población. Se podría llegar a afirmar que el sector se vio «empujado» a utilizar este tipo de técnicas por la presión que ejercieron los propios participantes en las investigaciones, que estaban respondiendo las encuestas que les llegaban utilizando sus teléfonos móviles, incluso cuando las encuestas no estaban optimizadas para ser respondidas a través de estos dispositivos (Ochoa y Castro, 2015; White y Stevens, 2015). El porcentaje de respuestas a encuestas con diseños no responsivos que eran completadas vía móvil llegó a superar en algunos estudios el 40\% (Murphy, 2015; Wells, 2015). Esta tendencia, que tuvo lugar sobre todo entre los años 2010 y 2014, se denominó investigación móvil «por accidente» 0 «inintencionada» (inintencionada por parte del investigador) (Peterson, 2013) y fue un motivo importante de preocupación para el sector, que se vio forzado a diseñar cuestionarios responsivos, platfom agnostic, a través de los cuales el investigador facilitaba al participante la mayor variedad de opciones posibles para responder.

En cualquier caso, el interés del sector de la investigación de marketing por las metodologías móviles se ha basado en los siguientes aspectos: la creciente ubicuidad de los dispositivos móviles; la aparición de nuevos y más potentes terminales; y el desarrollo de nuevos enfoques de investigación del consumidor, facilitando las investigaciones en tiempo real y la posibilidad de recoger datos de forma pasiva, así como fomentando la colaboración más activa de los participantes en los estudios. En los siguientes apartados se desarrollan estos aspectos y se explican las principales ventajas y limitaciones de la investigación de marketing en dispositivos móviles. También se muestran los datos sobre el nivel de uso de las metodologías móviles en la industria y se presentan los resulta-

1. El tabléfono (alternativa en español al neologismo inglés phablet) es un dispositivo electrónico, híbrido de teléfono inteligente y tableta, con una pantalla táctil de cinco a siete pulgadas.

2. La tecnología ponible (wearable technology en inglés) es la que incorporan prendas de vestir, relojes, gafas y otros complementos personales que se suelen llevar encima. Actualmente, la ropa y los complementos con mayor difusión son los relojes inteligentes, las pulseras de actividad, las gafas inteligentes, las videocámaras, los auriculares de realidad virtual y la ropa inteligente. 
dos de una investigación doctoral que analiza los factores que han influido en la «lenta» adopción de las técnicas móviles por parte de la industria de la investigación de mercados en España.

\section{Por qué resulta interesante la investigación móvil}

Una de las principales razones por las que la investigación móvil resulta interesante para la industria es por la creciente ubicuidad de los dispositivos móviles (AAPOR, 2014; Poynter et al., 2015). En la actualidad, casi todos disponemos de un dispositivo móvil, y lo llevamos con nosotros prácticamente a cualquier lado, formando parte de nuestra cotidianidad. Los datos de penetración de los teléfonos móviles inteligentes, y de otros dispositivos móviles, como las tabletas, cambian continuamente. Aun así, y con el objetivo de mostrar la dimensión del fenómeno móvil, tomemos en consideración los siguientes datos del informe elaborado por Ditrendia (2018) y el de Gartner (2018):

- En 2017 se vendieron en el mundo 1.500 millones de teléfonos móviles inteligentes.

- A nivel mundial, en 2018 había 5.135 millones de usuarios de teléfonos móviles, lo cual supone el 68\% de la población. Entre estos, más de 3.700 millones de usuarios tenían acceso a internet en su móvil. En España este porcentaje era del $80 \%$ (29 millones de usuarios), ocupando el sexto lugar en el ranking mundial.

- El teléfono móvil es el dispositivo más usado para acceder a internet, en detrimento del ordenador de sobremesa o del portátil; lo utilizan el $97 \%$ de los usuarios.

- También es el primer dispositivo al que se recurre para casi todas las necesidades. En 2017, el número de usuarios «solo móvil» frente a los que combinan ordenador y móvil se ha incrementado en casi todos los países del mundo. En España, el 32\% de la población usuaria de móvil únicamente utiliza esta plataforma para acceder a internet.

Por otra parte, los nuevos y cada vez más potentes terminales han permitido ampliar el uso de estos dispositivos en investigaciones de marketing (Callegaro et al., 2015). Los primeros estudios de mercado con dispositivos móviles se realizaron a principios de la década de 1990 a partir de mensajes cortos de texto (SMS), mediante aplicaciones WAP, o a través de software descargado en los terminales (como aplicaciones con lenguaje JAVA). Estas opciones tenían una limitación técnica y frecuentemente requerían de un alto grado de colaboración del participante en la investigación. En contraste, los mayores tamaños de las pantallas de los teléfonos móviles, tabléfonos y tabletas, así como las nuevas velocidades de conexión a internet (3G, 4G, 5G, wifi...) han posibilitado la existencia de un mayor número de investigaciones que pueden ser conducidas vía móvil. De igual manera, la estandarización de los procesos de creación de aplicaciones (apps), y la facilidad de descarga de estas desde las app stores han facilitado el desarrollo de nuevas herramientas tecnológicas para la captación de información del comportamiento del consumidor.

Otro motivo por el que la investigación de marketing con dispositivos móviles despierta interés en el sector es por la posibilidad de llevar a cabo estudios más cerca del «momento de la verdad». Existen evidencias de que las investigaciones que tienen lugar in-the-moment (como cuando alguien está comprando en una tienda, comiendo en un restaurante o alojado en un hotel) proporcionan información más profunda, precisa y veraz de los consumidores respecto a la que se conseguiría mediante estudios conducidos con posterioridad a estos actos de compra o consumo (Poynter et al., 2014; Wells et al., 2014; Wells, 2015). Y ello porque la información que se pretende recoger está aún reciente en la mente de las personas. Precisamente, la constante disponibilidad de los dispositivos móviles, especialmente de los teléfonos, permite a los investigadores llevar a cabo estudios más cercanos al «momento de la verdad», próximos a la experiencia de consumo. Sin embargo, la investigación de mercados más tradicional ha venido desarrollando estos estudios días después, o incluso semanas o meses después, de que tuviera lugar la interacción del consumidor con los productos, servicios o anuncios que quieren ser testados, pudiéndose llegar a producir lo que se denomina «sesgo de memoria».

Los dispositivos móviles también permiten realizar investigaciones con sistemas de recogida pasiva de datos. Los terminales móviles, principalmente los teléfonos inteligentes y las tabletas, pueden recopilar una gran variedad de información mientras que el participante en una investigación de mercado desarrolla su actividad cotidia- 
na, y sin solicitar su participación activa (Poynter, 2015). En la mayoría de los casos, este ha debido descargarse una app en su teléfono, a través de la cual se puede, por ejemplo, hacer un seguimiento del uso que hace de su terminal para navegar por internet, comprar, consultar cuentas bancarias, ver películas en línea, etc. Estas investigaciones se realizan bajo enfoques cualitativos y cuantitativos, y pueden llevarse a cabo de forma combinada con la aplicación de sistemas de geolocalización. Los dos usos clave de los sistemas de geolocalización son el etiquetado (tagging) -por ejemplo, registrando la ubicación en tiempo real de una persona- y el geovallado (geofacing) -por ejemplo, enviando una encuesta al teléfono móvil de la persona cuando entra o sale de una determinada ubicación, como puede ser una tienda específica (Drew y Barney, 2015)-. En las investigaciones con datos de geolocalización, las encuestas basadas en la ubicación requieren que el participante proporcione su localización en una zona geográfica o de geovallado predefinida. Al entrar, salir o simplemente pasar una cantidad de tiempo predeterminada en esa zona definida, se activa un trigger-procedimiento que se ejecuta cuando se da una condición establecida- y se le envía una encuesta a su dispositivo móvil. Se recurre a este tipo de estudios para medir la satisfacción sobre la experiencia de visita a un establecimiento o la efectividad de una campaña de comunicación o para promocionar empresas o marcas.

Otra tipología de estudios que los dispositivos móviles facilitan son las investigaciones de carácter etnográfico o las desarrolladas mediante comunidades en línea de investigación de mercado, dos de las técnicas más empleadas a la hora de llevar a cabo investigaciones en las que se solicita la colaboración activa de los participantes en la investigación. Este tipo de investigaciones hace posible el estudio en profundidad de los hábitos y comportamientos de los individuos y a menudo implica el uso de dispositivos móviles para que registren vídeos, tomen fotografías, escriban mensajes de texto, etc., proporcionando un feedback rápido o respuestas más detalladas a las solicitudes de información de los investigadores.

Además de los beneficios señalados, la investigación de marketing mediante dispositivos móviles presenta otras ventajas respecto al resto de técnicas, que parten de los beneficios habituales de la investigación de marketing en línea: el ahorro en costes y la rapidez en la obtención de datos (Malhotra y Peterson, 2001; Schonlau et al., 2001; llieva et al., 2002; Zikmund, 2003). De hecho, las encuestas móviles suelen ser respondidas minutos después de ser recibidas (Callegaro, 2010; Macer, 2010; Buskirk y Andrus, 2012). Asimismo, gracias a la tecnología móvil, se alcanzan poblaciones objetivo difícilmente accesibles a través de métodos tradicionales y otras formas de encuestas en línea, como los consumidores residentes en países en vías de desarrollo o los millennials (Robbins, 2011; Drew y Barney, 2015).

\section{Algunas limitaciones de la investigación móvil}

En contrapartida, entre las limitaciones de las técnicas móviles está la necesidad de elaborar cuestionarios breves, sencillos y optimizados para pantallas móviles, ya que de lo contrario puede incrementarse la posibilidad de abandono del cuestionario y, por lo tanto, disminuir la tasa de respuesta y la calidad de los datos obtenidos en el estudio (Mavletova, 2013; Callegaro et al., 2015; Antoun, 2015). En este sentido, el menor tamaño de las pantallas de los terminales móviles y la menor variedad de formatos de preguntas que admiten podrían suponer una limitación importante a la hora de dar respuesta a los objetivos de una investigación determinada, por lo que se desaconseja su uso en el caso de cuestionarios largos y/o complejos (Mavletova y Couper, 2013; Couper, 2013; Antoun, 2015).

Por otro lado, existen evidencias de diversos estudios que comparan los datos proporcionados en cuestionarios contestados en dispositivos móviles frente a los obtenidos en cuestionarios en línea respondidos en ordenador de sobremesa o portátil. Aunque la mayor parte de estas evidencias no señalan diferencias significativas entre las respuestas proporcionadas en diferentes dispositivos (De Bruijne y Wijnant, 2013; Mavletova y Couper, 2013; Wells et al., 2014), sí que sostienen, por ejemplo, que en los cuestionarios móviles las respuestas a las preguntas abiertas son más cortas (Callegaro et al., 2015). Respecto al sesgo de deseabilidad social los resultados son contradictorios, aunque potencialmente existe un mayor sesgo de este tipo en los cuestionarios móviles debido a la existencia de un menor sentido de privacidad -por ejemplo, si se está participando en el estudio desde un espacio público (Poyter et al., 2014)-. Sobre este último aspecto, los profesionales argumentan que en la investigación 
móvil pueden llegar a ser preocupantes aspectos de seguridad, éticos o de privacidad. Por esto en estudios con dispositivos móviles se debe asegurar que no se solicita nada ilegal (fotografiar personas sin permiso o en lugares donde no está permitido) y que no se pone en riesgo la vida de los participantes (contestar encuestas mientras se conduce, por ejemplo). Además, especialmente en estudios donde se recogen datos de forma pasiva, el participante debe estar informado del proceso y haber otorgado su consentimiento.

Otros aspectos a considerar, que podrían suponer barreras al uso de este tipo de metodologías móviles, serían la percepción de posibles costes extras (al realizar llamadas a teléfonos móviles, por ejemplo), las dificultades para controlar las cuotas de respuesta por regiones geográficas o los problemas derivados de combinar en un estudio muestras procedentes de líneas fijas y de líneas móviles (Poynter, 2015).

A lo largo de los últimos años las investigaciones en línea en las que los participantes utilizan un ordenador de sobremesa o un portátil se han optimizado en términos de velocidad en la recolección de datos y en coste (debido, sobre todo, al desarrollo de los paneles en línea) y, como veremos a continuación, continúan siendo la opción preferida, por encima de los estudios móviles, como metodología de investigación en estudios de mercado..

\section{Nivel de adopción de las metodologías móviles de investigación de marketing}

Esomar (European Society for Opinion and Marketing Research) publica anualmente un informe sobre el sector de la investigación de mercados, el Global Market Research. Entre los datos que se presentan en este informe, se encuentra el gasto por tipo de metodología de investigación. Por lo que respecta a la investigación móvil, esta información está disponible desde 2014 (anteriormente el gasto en la investigación móvil se computaba junto al de la investigación en línea).

Figura 1. Gasto por metodología de investigación utilizada (mundial) (\%)

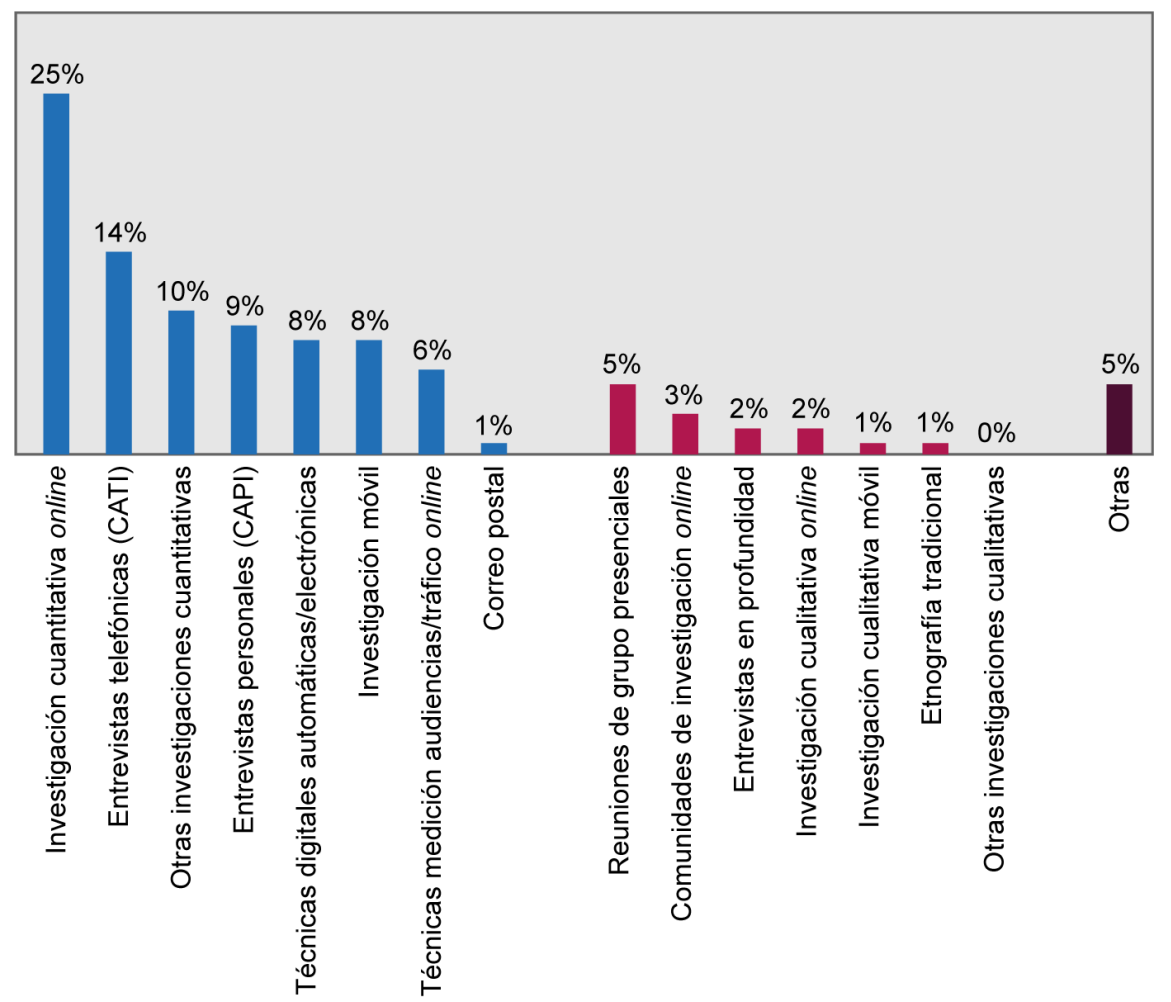

Fuente: Global Market Research (Esomar, 2018). 
La figura 1 recoge el porcentaje de gasto por metodología utilizada en el último año con datos publicados, el 2017. Se puede observar que la investigación cuantitativa (en azul) sigue siendo la principal protagonista en el sector, con el $81 \%$ del gasto total. La investigación cualitativa (en rojo) representa el 14\% del gasto, mientras que el 5\% restante lo conforman otras tipologías de investigación (como la recogida de información de fuentes secundarias).

En 2017, y como viene siendo habitual desde principios de la década de 2000, la investigación cuantitativa en línea es la metodología en la que se produce un mayor gasto (25\%), seguida por las encuestas telefónicas -aquí también se presenta, por primera vez, el gasto en encuestas realizadas a teléfonos móviles (14\%)-. Las encuestas presenciales representan el 9\% del gasto en investigación, mientras que la investigación realizada a través de dispositivos móviles solo alcanza el $8 \%$ del gasto total. Por lo que respecta a la investigación cualitativa, los grupos de discusión presenciales generan el mayor gasto (5\%), mientras que las comunidades de investigación en línea representan el 3\%. La investigación móvil cualitativa -en la que se incluyen principalmente estudios etnográficossupone el 1\% del gasto total. Si tenemos en cuenta el gasto total en investigación móvil, ya sea cuantitativa o cualitativa, este alcanza el 9\% en 2017 a nivel mundial. Esomar también ofrece este dato por país participante, y en el caso de España es del 1\%.

Si observamos a nivel mundial la evolución del porcentaje de gasto en las metodologías en línea y móvil desde el año 2014 (figura 2), vemos que durante los años 2014-2016 tanto el gasto en investigación en línea como en investigación móvil se ha incrementado (en porcentajes relativos mayores en el caso de la investigación en línea). Este hecho puede implicar que la investigación móvil no es una metodología de reemplazo de las técnicas en línea y que se ha venido utilizando en proyectos con objetivos de investigación diferentes o dirigidos a poblaciones distintas. Si tenemos en cuenta los datos de evolución del gasto proporcionados por Esomar para la industria española (figura 3), podemos observar que si bien el gasto en investigación en línea se mantiene estable durante los últimos años, la investigación móvil alcanza unos niveles de gasto muy bajos.

Figura 2. Gasto en investigación en línea y móvil (mundial) (\%)

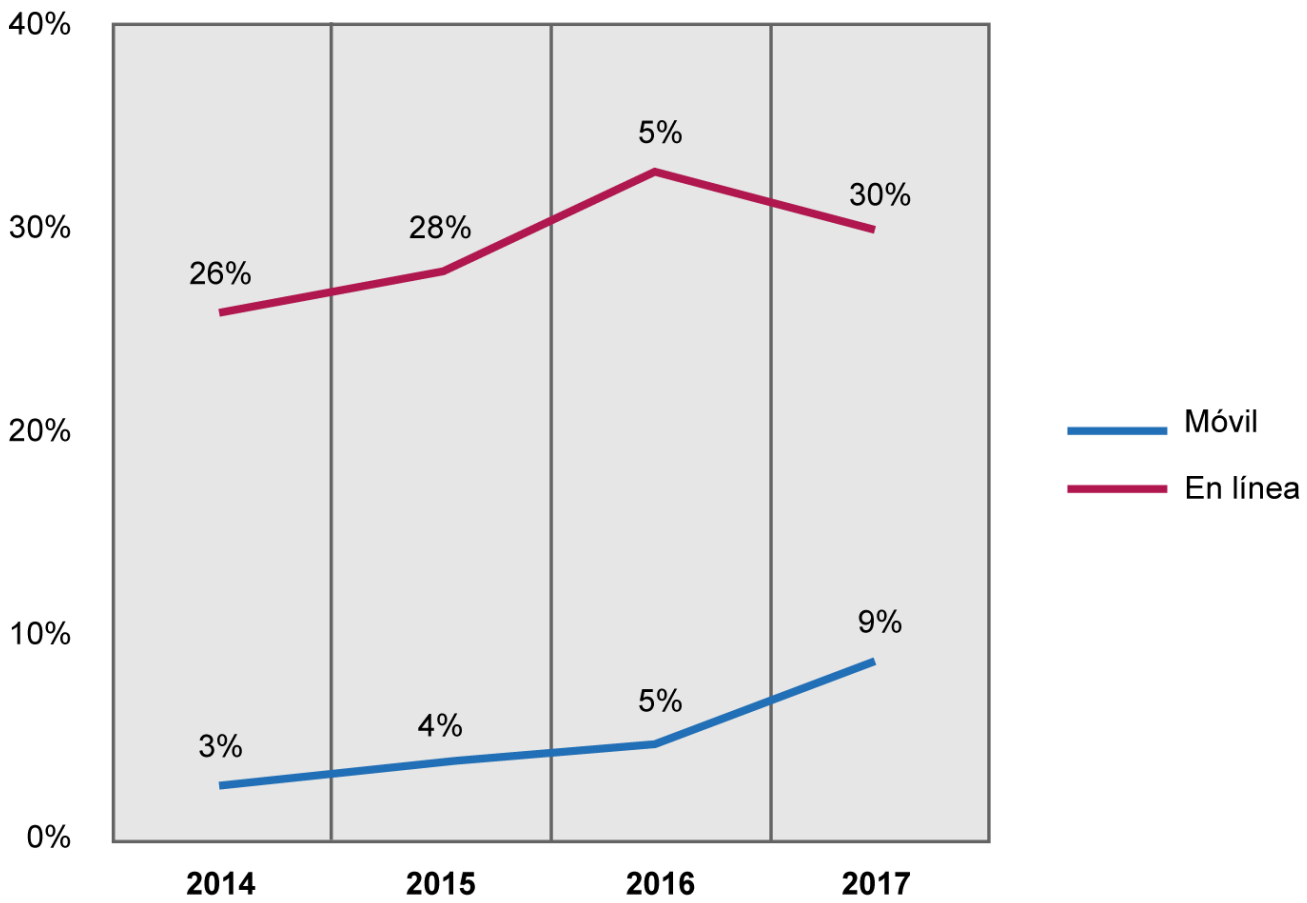

Fuente: elaboración propia a partir de Global Market Research (Esomar). 
Figura 3. Gasto en investigación en línea y móvil (España) (\%)

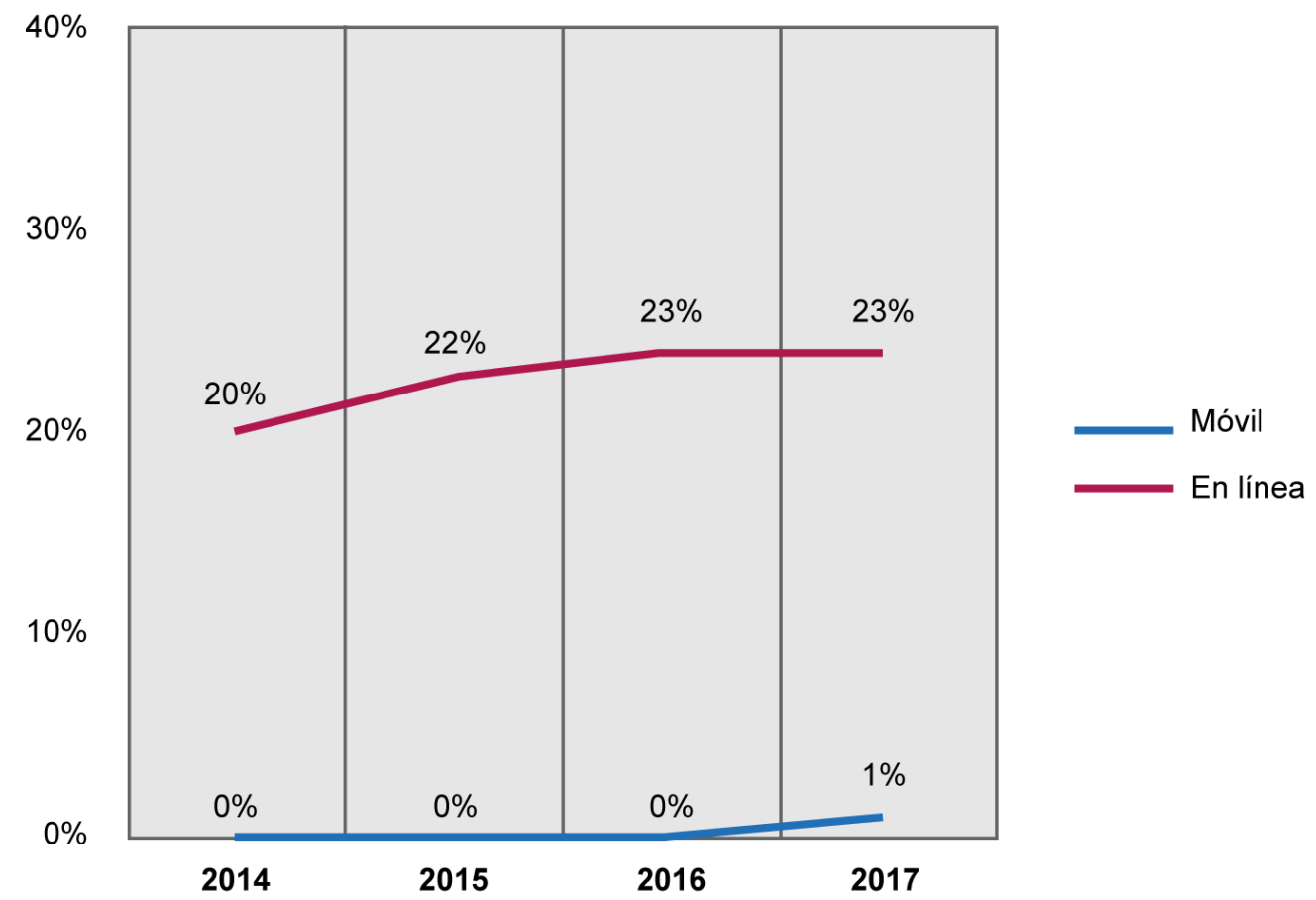

Fuente: elaboración propia a partir de Global Market Research (Esomar)

Sin embargo, en el último año registrado, 2017, se observa un cambio de tendencia en el gasto en ambas metodologías. A nivel mundial, el gasto en investigación en línea disminuyó en 3 puntos porcentuales (del 33\% al $30 \%$ ), mientras que la investigación móvil incrementó su gasto en 4 puntos porcentuales (del 5\% al 9\%). Aunque de forma absoluta el nivel de gasto en la investigación en línea sigue siendo muy superior, será necesario hacer un seguimiento de estos datos durante los próximos años para confirmar o no un cambio de tendencia en el uso de estas dos metodologías.

\section{Factores que afectan al bajo nivel de adopción y difusión de las metodologías móviles}

Al analizar los datos sobre el uso de la investigación móvil, cabe preguntarse qué factores han podido influir en el bajo nivel de adopción y difusión de las metodologías móviles entre las empresas de la industria de la investigación de mercados. Diversos informes del sector -elaborados por Esomar y Greenbook- y algunos investigadores académicos -como Chang y Krosnick (2009), Warta (2015) o White y Stevens (2015)- han apuntado a que la actitud conservadora de la industria, así como un perfil resistente a los cambios metodológicos característico de sus profesionales, junto con un alto nivel de satisfacción con las técnicas de investigación en línea, podrían llegar a explicar por qué a la investigación móvil le está costando tanto despegar.

Para profundizar en los factores determinantes del uso de la investigación móvil en la industria española de investigación de marketing e identificar aquellos elementos que más han podido influir en este lento ritmo de adopción, se ha llevado a cabo una investigación, enmarcada en una tesis doctoral, en la que han participado empresas del sector de la investigación de mercados español, muchas de ellas pertenecientes a la Asociación Española de Estudios de Mercado, Marketing y Opinión (AEDEMO). Las teorías de difusión de innovaciones 
(Rogers, 1983) y de aceptación tecnológica (Davis, 1989), así como los modelos de adopción de innovaciones tecnológicas, concretamente el modelo T-O-E (Tecnología-Organización-Entorno) (Tornatzky y Fleischer, 1990), adaptados al análisis de la adopción de la investigación de mercados móvil (figura 4), han ayudado a crear el marco teórico de la investigación doctoral y han facilitado la comprensión de los distintos elementos determinantes de la aceptación e intención de uso de la investigación móvil.

El modelo T-O-E presenta como determinantes del nivel de uso de una innovación tecnológica factores tecnológicos, organizativos y del entorno, los cuales permiten explicar el uso de dicha innovación a nivel empresarial. En la investigación se han tenido en cuenta, como variables del contexto tecnológico, los beneficios y limitaciones percibidas de la investigación móvil. Han formado parte del contexto organizacional variables relacionadas con el nivel de competencia profesional o expertise con los que cuenta la organización para adoptar las metodologías móviles, la actitud hacia el cambio, la satisfacción con técnicas tradicionales de investigación de marketing y la dimensión empresarial. Por su parte, las variables del entorno consideradas en el modelo han sido las presiones procedentes de los competidores, de los clientes de las compañías que contratan los estudios, así como la ejercida por los propios participantes en las investigaciones de mercado.

Figura 4. Modelo de adopción de la investigación de marketing con dispositivos móviles, basado en modelo T-O-E*

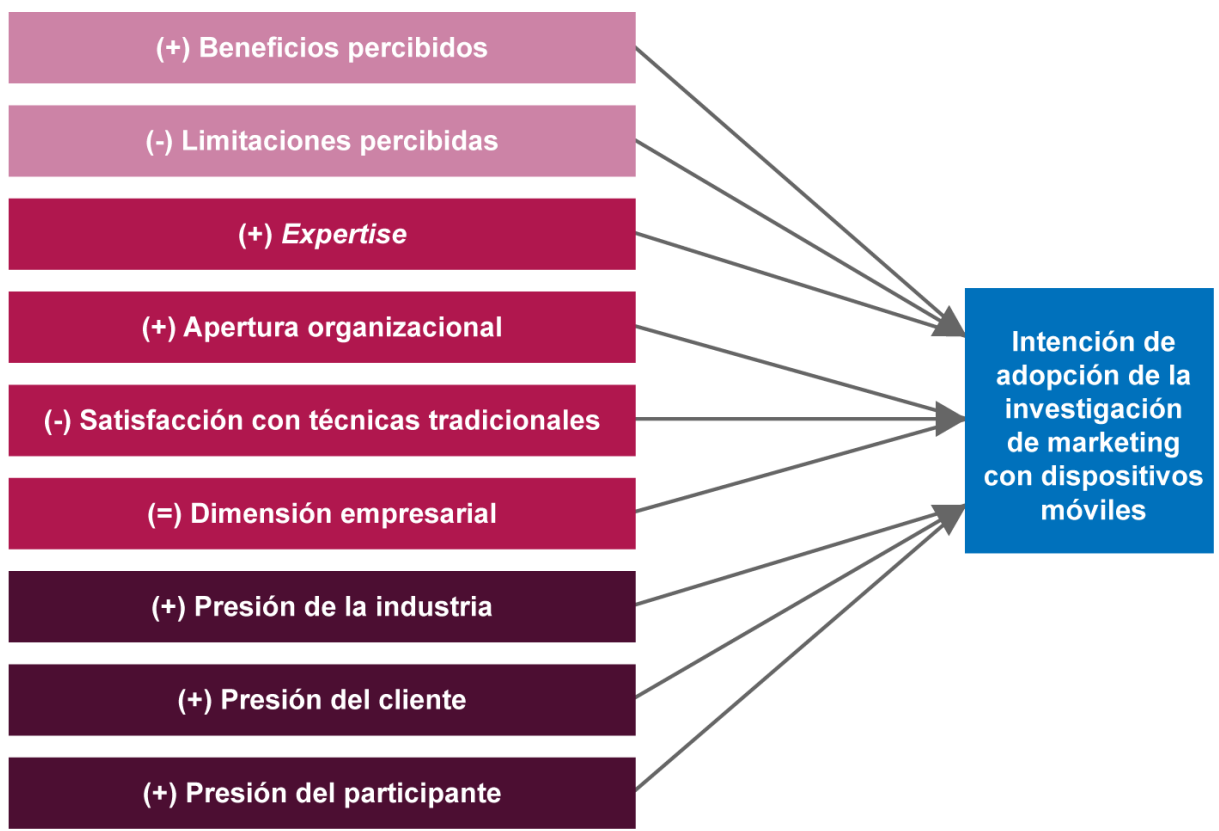

Fuente: elaboración propia.

*Entre paréntesis se indica el sentido de la relación (positiva o negativa) de cada variable independiente del modelo respecto a la variable dependiente. Para la variable "Dimensión empresarial", no se establece un sentido de la relación.

Los resultados obtenidos en esta investigación señalan que las ventajas y limitaciones asociadas a la metodología móvil no constituyen un factor significativo de influencia (ni positiva ni negativa, respectivamente) de su adopción y difusión. Sin embargo, sí que influye en la intención de uso de las metodologías móviles, de forma significativa y positiva, el poseer una actitud abierta hacia los cambios y las innovaciones, y contar con experticia dentro de la compañía. Por otra parte, las influencias o presiones que las organizaciones que se dedican a la investigación de marketing reciben de sus competidores, de los clientes que contratan sus investigaciones y, sobre todo, de los individuos que participan en los estudios constituyen elementos importantes de influencia -significativa y positivaen el uso de la investigación móvil. 
La investigación también demuestra que tener un alto nivel de satisfacción cuando se trabaja con técnicas tradicionales de investigación de marketing (como las entrevistas personales, telefónicas y, sobre todo, en línea) es un factor que ha ralentizado la adopción de la investigación móvil entre las compañías de la industria, ya que se constata una relación significativa negativa entre esta variable de satisfacción con técnicas tradicionales y la adopción de la investigación móvil. Por último, se ha comprobado que la variable dimensión empresarial, la cual tiene en cuenta el propio tamaño de la compañía (número de trabajadores), así como la posesión de otros elementos relacionados con ella (panel propio, si se trata o no de una compañía internacional), no constituye un factor determinante del uso de las metodologías móviles.

\section{Conclusiones}

Actualmente, las compañías del sector de la investigación de marketing están utilizando las metodologías móviles en sus estudios sobre la base de una mayor conveniencia para los participantes -a estos les resulta más fácil, cómodo y rápido participar a través de sus dispositivos móviles- y como elemento de diferenciación estratégica frente a sus competidores. A pesar de esto, y del interés que la industria de la investigación de marketing ha venido demostrando en los últimos diez años por las diferentes metodologías que emplean los dispositivos móviles como elementos de captación de datos de los participantes en los estudios, su uso y, especialmente, su difusión entre las compañías se han mantenido bastante limitados. Los factores que permiten explicar estos bajos niveles de adopción están relacionados con una actitud algo resistente a los cambios por parte de los profesionales del sector, así como con un alto nivel de satisfacción con otras metodologías tradicionales y, sobre todo, con la investigación desarrollada en línea, la cual se ha mantenido a lo largo de los últimos años como metodología predominante. Los últimos datos publicados por Esomar apuntan un tímido incremento en el gasto en las metodologías móviles en 2017. Será necesario consultar próximos informes para seguir su evolución y confirmar, o no, que estamos ante un cambio de tendencia respecto al uso de metodologías de investigación de marketing por parte del sector.

\section{Referencias bibliográficas}

AAPOR (American Association for Public Opinion Research) (2014). Mobile technologies for conducting, augmenting and potentially replacing surveys: report of the aapor task force on emerging technologies in public opinion research. Deerfield, IL: American Association for Public Opinion Research.

AEDEMO (Asociación Española de Estudios de Mercado y Opinión). <http://www.aedemo.es>.

ANTOUN, C. (2015). "Mobile web surveys: a first look at measurement, nonresponse, and coverage errors». Dissertation University of Michigan [disponible en línea]. <www.lissdata.nl/dataarchive/hosted_files/download/3106>.

BUSKIRK, T. D.; ANDRUS, C. (2012). «Smart surveys for smart phones: exploring various approaches for conducting online mobile surveys via smartphones». Survey Practice. Vol. 5.

CALLEGARO, M. (2010). «Do you know which device your respondent has used to take your online survey?». Survey Practice, 3 [disponible en línea]. <http://www.surveypractice.org/index.php/surveypractice/article/view/250/ html>.

CALLEGARO, M.; LOZAR MANFREDA, K.; VEHOVAR, V. (2015). Web Survey Methodology. Londres: SAGE.

DAVIS, F.D. (1989). «Perceived usefulness, perceived ease of use, and user acceptance of information technology». MIS Quarterly. Vol. 3, n.ำ13, págs. 319-340.

DE BRUIJNE, M.; WIJNANT, A. (2013). «Comparing survey results obtained via mobile devices and computers: an experiment with a mobile web survey on a heterogeneous group of mobile devices versus a computer-assisted web survey». Social Science Computer Review. Vol. 31, págs. 482-504. 
DITRENDIA (2018). Informe ditrendia: Mobile en España y en el mundo 2018 [disponible en línea]. <https://ditrendia.es/informe-mobile-2018/>.

DREW. M.; BERNEY, P. (2015). «What does mobile really mean for research?». Journal of direct, data and digital practice. Vol. 17, n. ${ }^{\circ}$ 1, págs. 14-19.

ESOMAR (European Society for Opinion and Marketing Research) (2018a). Guideline for Conducting Mobile Market Research [disponible en línea]. <http://www.esomar.org>.

ESOMAR (European Society for Opinion and Marketing Research) (2018b). Global market research 2018. <http:// www.esomar.org $>$.

GARTNER (2018). «Market share: pcs, ultramobiles and mobile phones, all countries, 2Q18 Update» (Agosto 2018) [disponible en línea]. <https://www.gartner.com/doc/3888165/market-share-pcs-ultramobiles-mobile>.

ILIEVA, J.; BARON, S.; HEALEY, N.M. (2002). «Online surveys in marketing research: pros and cons». International Journal of Market Research. Vol. 44, n. ${ }^{\circ}$ 3, págs. 361-382.

MACER, T. (2010). «Mobile research: too much excitement or not enough?». Quirk's marketing research review.

MALHOTRA, N.K.; PETERSON, M. (2001). "Marketing research in the new millennium: emerging issues and trends». Marketing Intelligence \& Planning. Vol. 19, n. 4, págs. 216-235.

MAVLETOVA, A. (2013). «Data quality in pc and mobile web surveys». Social Science Computer Review. Vol. 31 , págs. 725-743.

MAVLETOVA, A.; COUPER, M.P. (2013). «Sensitive topics in pc web and mobile web surveys: is there a difference?». Survey Research Methods. Vol. 7, págs. 191-205.

MAXL, E. (2009). «Mobile market research: analysis through the mobile phone». En: Maxl, E.; Doering, N.; Wallisch, A. (eds.). Mobile Market Research (págs. 11-39). Colonia, Alemania: Herbert Von Halem.

MURPHY, L. (ed.). (2015). Greenbook Research Industry Trends (GRIT) Report [disponible en línea]. <http://www. greenbook.org/grit>.

MURPHY, L. (ed.). (2018). Greenbook Research Industry Trends (GRIT) Report [disponible en línea]. <http://www. greenbook.org/grit>.

PETERSON, G. (2013). «What can we learn from unintentional mobile respondents». Casro Journal 2012-13. págs. 32-35 [disponible en línea]. <http://www.websm.org/uploadi/editor/1388761460Peterson_2012_What_ We_Can_Learn.pdf>.

OCHOA, C.; CASTRO, V. (2015). «Investigación móvil: ¿al servicio del investigador o del investigado?». Investigación y Marketing. N. ${ }^{\circ} 128$, págs. 42-47.

POYNTER, R.; WILLIAMS, N.; YORK, S. (2014). The handbook of mobile market research. Tools and techniques for market researchers. Reino Unido: Wiley.

POYNTER, R. (2015). «The utilization of mobile technology and approaches in commercial market research». En: Mobile research methods: opportunities and challenges of mobile research methodologies. Londres: Ubiquity Press.

POYNTER, R.; TONINELLI, D.; DE PEDRAZA, P. (2015). «Mobile Research Methods: Possibilities and Issues of a new promising way of conducting research». En: Mobile research methods: opportunities and challenges of mobile research methodologies. Londres: Ubiquity Press.

ROBBINS, C. (2011). Mobilizing market research [disponible en línea]. <http://www.greenblog.org/mobile-research>. ROGERS, E. M. (1983). Diffusion of innovations. Nueva York: Free Press.

SCHONLAU, M.; FRICKER, R. D.; ELLIOTT, M. N. (2001). Conducting research surveys via e-mail and the web. Santa Monica, California: Rand Corporation.

TONINELLI, D. (ed.); PINTER, R.; DE PEDRAZA, P. (2015). Mobile research methods: opportunities and challenges of mobile research methodologies. Londres: Ubiquity press.

TORNATZKY, L.G.; FLEISCHER, M. (1990). The processes of technological innovation. Lexington, Massachusetts: Lexington Books.

TOURANGEAU, R. (2004). «Survey research and societal change». Annual Review of Psychology. Vol. 55, págs. 775-801.

VENKATESH, V.; MORRIS, M.G.; DAVIS, G.B.; DAVIS, F.D. (2003). «User acceptance of information technology: toward a unified view». MIS Quarterly. Vol. 27, n. ${ }^{\circ}$ 3, págs. 425-478. 
WARTA, M. (2015). «The market research industry: early adopter or laggard?» En: Grit Report 2015 Q3 - Q4, pág. 23.

WELLS, T.; BAILEY, J.T.; LINK, M.W. (2014). «Comparison of smartphone and online computer survey administration». Social Science Computer Review. Vol. 32, págs. 238-255.

WELLS, T. (2015). «What market researchers should know about mobile surveys». International Journal of Market Research. Vol. 57, n. ${ }^{\circ}$ 4, págs. 521-532.

WHITE, D.; STEVENS, C. (2015). «Consumers have gone mobile long ago - researchers must catch up before it's too late». Research World [disponible en línea]. <https://rwconnect.esomar.org/consumers-have-gonemobile-long-ago-researchers-must-catch-up-before-its-too-late/>.

ZIKMUND, W.G. (2003). Fundamentos de investigación de mercados. Madrid: Thomson.

Cita recomendada: PACHECO BERNAL, Carmen. La investigación de marketing con dispositivos móviles: un lento despertar. Oikonomics [en línea]. Mayo 2019, no. 11, pp. 43-53. ISSN: 2339-9546. DOI: https://doi.org/10.7238/o.n11.1906

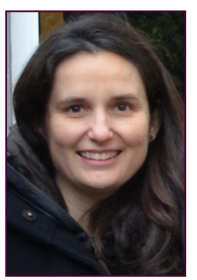

\section{Carmen Pacheco Bernal}

mpachecob@uoc.edu

\section{Directora del grado en Marketing e investigación de mercados (UOC)}

Licenciada en Investigación y Técnicas de Mercado y diplomada en Ciencias Empresariales por la Universidad de Barcelona; Diploma de Estudios Avanzados por la UOC y doctoranda del Programa de Doctorado en Sociedad de la Información y el Conocimiento de la misma universidad. Es profesora de los Estudios de Economía y Empresa de la UOC desde 2004, donde coordina asignaturas del ámbito del marketing y de la investigación de mercados. Desde 2014 ejerce el cargo de directora de Programa del Grado de Marketing e Investigación de Mercados. Anteriormente, su experiencia profesional estuvo vinculada a la consultoría de investigación de mercados. Su actividad de I+D+i se sitúa principalmente en el campo del marketing digital y, más concretamente, en la investigación de marketing en línea y móvil. Además, ha desarrollado actividad de investigación en el ámbito de la formación virtual en el puesto de trabajo y en el de la evaluación de impactos económicos, sociales y ambientales de eventos turísticos. Es miembro del grupo de I+D+i DigiBiz (Digital Business Research Group) de la UOC.

Los textos publicados en esta revista están sujetos -si no se indica lo contrario- a una licencia de Reconocimiento 4.0 Internacional de Creative Commons. Puede copiarlos, distribuirlos, comunicarlos públicamente, hacer obras derivadas siempre que reconozca los créditos de las obras (autoría, nombre de la revista, institución editora) de la manera especificada por los autores o por la revista. La licencia completa se puede consultar en https://creativecommons.org/licenses/by/4.0/deed.es_ES.

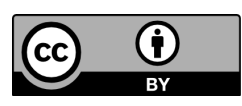

